\title{
Factor X Inactivation
}

National Cancer Institute

\section{Source}

National Cancer Institute. Factor X Inactivation. NCI Thesaurus. Code C131667.

Presence of inactivating antibodies to factor $\mathrm{X}$ in the blood. 